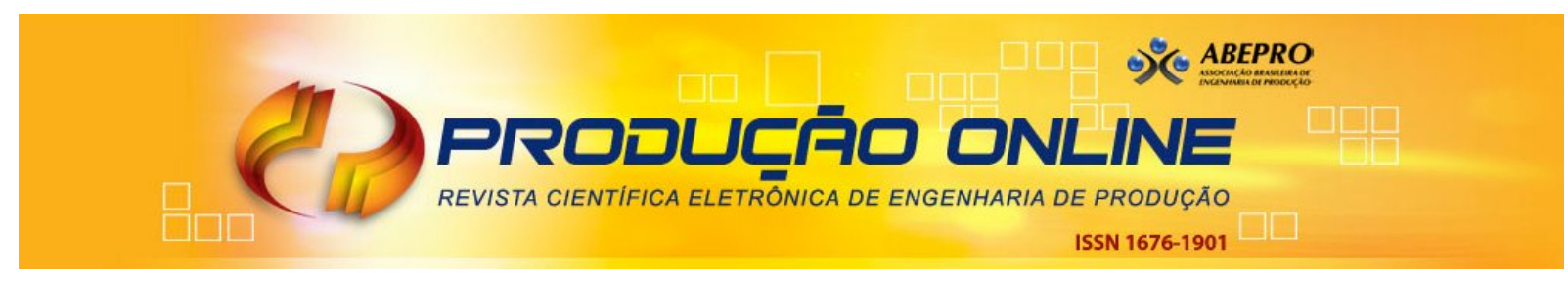

\title{
APLICAÇÃO DA MODELAGEM E SIMULAÇÃO NOS SISTEMAS DE FILAS M/M/S- ENTENDENDO A NATUREZA DA SIMULAÇÃO
}

\section{APPLICATION OF MODELING AND SIMULATION IN M/M/S QUEUE SYSTEM- UNDERSTANDING THE NATURE OF SIMULATION}

\author{
Marina Meireles Pereira* E-mail: eng.marinameireles@gmail.com \\ Maria Jose Pereira Dantas*E-mail: mipdantas@gmail.com \\ **Pontifícia Universidade Católica de Goiás (PUC/GO), Goiânia, GO
}

\begin{abstract}
Resumo: A Simulação se tornou uma abordagem de estudo utilizada em aplicações diversas, dentro da Engenharia. A simulação é a representação do comportamento de um processo real por meio de um modelo experimental, com o objetivo de avaliar o funcionamento deste e buscar melhorias em seu desempenho. A aplicação da simulação é de grande interesse se o problema em questão não é tão simples e requer uma modelagem mais realista. Este artigo propõe uma discussão que tem como pano de fundo a comparação entre Métodos Analíticos e Métodos Numéricos. Os métodos analíticos são aplicados a filas do tipo M/M/s com o objetivo de demonstrar aos alunos de Engenharia, de maneira intuitiva e razoavelmente simples e rápida, que as soluções obtidas pela abordagem de simulação são aproximadas e mais confiáveis quando se entende a natureza da simulação. A parte experimental utiliza o software Microsoft Excel para demonstrar questões importantes, como o tempo de aquecimento para obtenção do regime estável e número de replicações da simulação. São mostrados experimentos em que os valores da simulação convergem para os valores teóricos somente após um tempo grande de simulação. E que as replicações sempre apresentam variabilidade e os resultados de saída devem ser tratados estatisticamente.
\end{abstract}

Palavras-chave: Simulação. Filas. Métodos Analíticos. Método Numérico. Excel.

Abstract: Simulation becomes a study approach used in various applications, in engineering. Simulation is the behavior representation of a real process through an experimental model in order to evaluate its operation and seek improvements in its performance. The application of simulation is of great interest if the issue in question is not so simple and requires a more realistic modeling. This article proposes a discussion that has as background the comparison of Analytical Methods versus Numerical Methods. The analytical methods are applied to M/M/s queue aiming to show the Engineering students that, in intuitive, fairly simple and quick way, the solutions obtained by the simulation approach are approximate and more consistent when we understand the nature of the simulation. The experimental part uses the Microsoft Excel software to demonstrate important issues such as the warm-up time to obtain the steady state and the simulation replication number. The experiments showed that the simulation values converge to the theoretical values only after a very large simulation time. And further, the replications always present variability, and that the output results should be treated statistically.

Keywords: Simulation. Queue. Analytical Methods. Numerical Method. Excel.

\section{INTRODUÇÃO}

A simulação é uma técnica de estudo que vem sendo amplamente utilizada nas mais diversas áreas de estudo das Engenharias, devido ao desenvolvimento dos 
recursos computacionais, que estão cada vez mais capazes de avaliar cenários de crescentes complexidades sem dificultar o seu manuseio (PRADO, 2008; CHWIF e MEDINA, 2010). Muitos softwares de simulação podem ser utilizados, como por exemplo, ARENA, PROMODEL, FLEXSIM, entre outros.

A simulação computacional fornece à administração/gestão de serviços, segundo Fitzsimmons \& Fitzsimmons (2010), um laboratório experimental, que possibilita o estudo do modelo de um sistema real, determinando como esse sistema pode reagir às mudanças políticas, de níveis de recursos ou às variações na demanda de clientes.

Algumas das aplicações da simulação computacional podem ser encontradas nos trabalhos de Lima et al. (2016a), em que busca apresentar a aplicação do Mapeamento do Fluxo de Valor, associado à simulação, a fim de analisar os impactos da adoção do Lean Manufacturing no desempenho de uma empresa calçadista. Pereira \& Costa (2012) aplicaram a simulação a fim de desenvolver um modelo de simulação para auxiliar na tomada de decisões sobre a programação da produção de um frigorífico de peixe, e Baptista e Rangel (2013) desenvolveram a simulação com o objetivo de integrar e comunicar via rede um modelo de simulação a eventos discretos de duas vias semaforizadas, a um sistema de controle automático, operado em tempo real por um controlador lógico programável.

Uma das aplicações dos estudos da simulação é no sistema de filas de espera. As filas estão presentes em diversas situações e são formadas devido a um descompasso entre a demanda dos clientes e a capacidade de atendimento do sistema. Estes descompassos podem gerar perda de receita para as organizações, conforme Carneiro (2008). Na maioria dos casos reais é impraticável a oferta de uma infraestrutura que seja suficiente para atender a procura dos usuários. (ABENSUR et al., 2003).

Segundo Law (2007), algumas vantagens apresentadas pela simulação são responsáveis pela sua propagação como a possibilidade de descrever com maior precisão um sistema complexo real, estimar o desempenho de um sistema, realizar experimentos, avaliar novas propostas, entre outras.

No entanto, para que a simulação possa descrever com precisão um sistema real, é necessário, segundo Norato e Duarte (2011), um esforço na avaliação de dados de saída da simulação, para proporcionar uma análise adequada dos resultados. É indispensável o entendimento da natureza da simulação que lida com aleatoriedade e Revista Produção Online, Florianópolis, SC, v. 17, n. 1, p. 49-75, jan./mar. 2017. 
variabilidade. Os autores apresentam questões sobre a definição correta do tempo de aquecimento para a estabilização do sistema, seguida de diversas replicações para avaliação estatística do comportamento do modelo.

Ingolfsson e Grossman (2002) afirmam que a simulação de sistemas de filas por planilhas pode ser usada para ensinar aos alunos sobre a natureza das filas e apoiar a aprendizagem ativa, em que os alunos possam articular para si a causa de comportamentos de filas como: porque as filas se formam, por que persistem, o papel da variabilidade, e o efeito não-linear do aumento da utilização sobre o desempenho da fila.

Para a elaboração do artigo inicialmente foi realizada a simulação de um sistema de fila simples, de um único canal de atendimento (M/M/1), para posteriormente ser realizada a simulação de um sistema de fila simples com dois canais de atendimento (M/M/2).

Para ambas as simulações o processo de chegada e o processo de atendimento seguem a distribuição Exponencial e a disciplina das filas foi regida pelo first in first out (FIFO), na qual o primeiro cliente a entrar na fila é o primeiro cliente a ser atendido. Com o estudo, objetiva-se o entendimento da natureza da simulação computacional a partir da comparação dos resultados analíticos e simulados.

Desta forma o artigo está estruturado em cinco seções. A seção 2 apresenta uma revisão bibliográfica acerca de modelagem e simulação, que é o objeto de estudo, da teoria de filas e de seus modelos simulados neste estudo. A seção 3 expõe a metodologia do trabalho. A seção 4 apresenta o desenvolvimento da simulação e um paralelo entre seus principais resultados e considerações entre o modelo analítico e simulado. A seção 5 contém as conclusões do estudo.

\section{REVISÃO BIBLIOGRÁFICA}

\subsection{Modelagem e simulação}

A simulação de sistemas, segundo Chung (2004), é o processo de criar e experimentar um sistema por meio de um modelo matemático computadorizado. Este sistema pode ser definido como o conjunto de componentes ou processos que se interagem e que recebem entradas e oferecem resultados para algum propósito. 
De acordo com Prado (2008), a simulação é uma técnica que permite imitar o funcionamento de um sistema real e têm inúmeras aplicações no mundo atual em diversas áreas, que vão desde a produção em uma manufatura, o funcionamento de um banco, um pedágio, um porto, até o movimento dos papéis em um escritório.

$\mathrm{Na}$ área acadêmica algumas aplicações da simulação, que possuem o objetivo de estender o conhecimento em simulação, podem ser encontradas no trabalho de Bouzada (2010), que apresenta uma discussão teórica a respeito da dicotomia entre métodos analíticos e métodos numéricos, quando realizado a simulação de um sistema não tão simples, necessitando de uma modelagem mais realista. Outra aplicação pode ser encontrada no artigo de Artalejo e Lopez-Herrero (2007) em que, após uma revisão da literatura da cadeia de Markov, com ênfase no cálculo da distribuição no estado estacionário, utilizaram a simulação a fim de entender o comportamento da simulação de uma fila com vários servidores, de tempo discreto e com população finita, sob três políticas de filas diferentes, avaliando as principais medidas de desempenho.

Ingolfsson e Grossman (2002) visa por meio da simulação de sistemas de fila por planilhas destacar o desenvolvimento e uso de gráficos de simulação em tempo real para fornecer, de forma rica, meios visuais para a compreensão de filas e simulação, e técnicas para usar estes gráficos para motivar os alunos, melhorar a sua aprendizagem, e estimular o seu interesse na simulação. Para isso, os autores destacam as três abordagens para o ensino sobre filas que são a teoria analítica das filas, os pacotes de simulação dedicados, e a simulação por meio de planilhas eletrônicas.

Evans (2000) também apresenta em seu trabalho aplicações da simulação, na qual discute algumas questões-chave associadas a métodos de ensino de simulação utilizando planilhas do Excel, fornecendo exemplos ilustrativos da aplicação de simulação em planilhas. Para isso apresentando como foco a implementação, vantagens e limitações, discutindo tanto a simulação de Monte-Carlo, bem como a simulação de sistemas dinâmicos com a simulação de um sistema de filas com um único canal de atendimento (M/M/1).

Para reproduzir um sistema, cientificamente, é necessário definir um conjunto de hipóteses sobre como ele funciona, explicitando as relações matemáticas ou lógicas e constituindo o modelo do sistema. Desta forma, se as relações que compõem o modelo são simples, é possível utilizar métodos matemáticos (como álgebra, Revista Produção Online, Florianópolis, SC, v. 17, n. 1, p. 49-75, jan./mar. 2017. 
cálculo, ou teoria da probabilidade) para obter os resultados e informações exatas sobre o sistema, a chamada solução analítica. No entanto, como a maioria dos sistemas reais são complexos ao ponto de não permitir uma avaliação analítica, esses modelos devem ser estudados por meio da simulação (LAW, 2007).

Segundo Freitas Filho (2008) a simulação é uma excelente ferramenta de análise de sistemas mais realistas. Por outro lado, deve-se estar ciente que os resultados da simulação são, muitas vezes, de difícil interpretação devido à presença de múltiplos processos aleatórios no modelo. Law (2007), enfatiza que não se pode considerar os dados de saída de uma única replicação do sistema como verdade absoluta e desconsiderar o período de aquecimento (período em que os dados da simulação apresentam elevada variabilidade em relação aos dados do período estacionário), se o comportamento do estado estacionário do sistema é o que importa.

\subsection{Teoria de filas}

A Teoria de Filas é o estudo da espera em diversas formas de filas e utiliza do "modelo de filas" para representar os variados tipos de "sistema de filas" reais segundo Hillier e Lieberman (2013). De acordo com Prado (2008), a Teoria das Filas fornece modelos analíticos que resolvem o problema através de fórmulas matemáticas.

Segundo Hillier e Lieberman (2013) o processo básico dos modelos de fila é regido de forma que os "clientes" que necessitam de atendimento são gerados no tempo por uma "fonte de entradas", entrando no "sistema de filas" e esperando na "fila", caso necessário. De tempos em tempos, um integrante é selecionado para o atendimento por alguma regra estabelecida na "disciplina da fila". O cliente então é atendido pelo "mecanismo de atendimento" e após a conclusão do serviço deixa o sistema de filas.

Para Brandão et al. (2012), alguns elementos em conjunto determinam o sistema de filas com os clientes, a fila e o canal de atendimento, e este sistema de filas pode ser caracterizado por três componentes afirma Law (2007):

a) Processo de Chegada: consiste em descrever como os clientes chegam ao sistema, ou seja, a taxa de chegada de clientes $(\lambda)$;

b) Mecanismo de serviço: é determinado pela especificação do número de servidores (s), explicitando se cada servidor tem sua própria fila ou há uma fila comum para todos os servidores e pela distribuição de probabilidade do tempo Revista Produção Online, Florianópolis, SC, v. 17, n. 1, p. 49-75, jan./mar. 2017. 
de atendimento do cliente. O mecanismo de serviço é medido pela taxa/nível de serviço de um servido $(\mu)$;

c) Disciplina da Fila: consiste na regra que o servidor utiliza para escolher o próximo cliente da fila (se houver) quando o servidor completa o serviço do cliente atual podendo ocorrer segundo as regras: Fist In, First Out - FIFO; Last In, First Out - LIFO e Prioridade (os clientes são atendidos por ordem de importância ou com base em suas necessidades de serviço).

Bronson (1985) apud Brandão et al. (2012) ainda citam a capacidade do sistema como um componente característico das filas, pois é a capacidade do sistema que determina o número máximo de usuários no sistema, sejam os que estão sendo atendido ou os que estão esperando atendimento, podendo ser de capacidade finita ou infinita.

Desta forma, diante da diversidade de possibilidades de filas, a Notação de Kendall-Lee é a forma de descrever os sistemas de forma simplificada, a partir da utilização de cinco características sendo $A / B / c / D / E$, em que $A$ se refere ao tipo de distribuição de probabilidade do processo de chegada; B refere ao tipo de distribuição de probabilidade do processo de atendimento; c representa o número de postos de atendimento; $\mathrm{D}$ representa a capacidade física do sistema e $\mathrm{E}$ define a disciplina da fila. (FOGLIATTI E MATTOS, 2007)

As distribuições de probabilidade, que representam as características A e B podem ser do tipo M (Markoviana), representando a distribuição exponencial; D, distribuição degenerada (tempo constantes); Ek, distribuição de Erlang de ordem k, e G, distribuição geral, podendo ser qualquer distribuição arbitraria, conforme Hillier e Lieberman (2013).

Deste modo Fogliatti e Mattos (2007) apresentam como objetivo da Teoria de Filas a definição de medidas de desempenho do sistema tanto pela perspectiva dos usuários, com a medição do tamanho médio de fila e os tempos médios de permanência na fila e no sistema, tanto pela perspectiva do servidor com a medição do tempo médio de atendimento e a taxa de ociosidade dos servidores.

\subsubsection{Modelo analítico M/M/s}

O modelo $\mathrm{M} / \mathrm{M} / \mathrm{s}$ é um dos modelos mais usados na teoria das filas e parte do pressuposto de que todos os tempos entre chegadas sejam distribuídos de forma Revista Produção Online, Florianópolis, SC, v. 17, n. 1, p. 49-75, jan./mar. 2017. 
independente e idêntica, de acordo com o processo de entrada de Poisson, e tempos de atendimento também distribuídos de forma independente e idêntica, segundo a distribuição exponencial. (HILLIER E LIEBERMAN, 2013).

O caso mais simples da fila M/M/s é a fila M/M/1, de acordo com Freitas Filho (2008), em que o processo de chegadas e o tempo de atendimento seguem o processo Markoviano e o algarismo 1 indica a presença de um único servidor. Os valores de $\lambda$ e $\mu$ representam: a taxa média de chegada de usuários por unidade de tempo e a taxa média de atendimento por unidade de tempo, respectivamente. São utilizados diretamente no cálculo da taxa de utilização do sistema $(\rho)$ como pode ser vista na Equação 1:

$$
\rho=\frac{\lambda}{\mu}
$$

Com base em Hillier e Lieberman (2013) a fila M/M/1 pode ser representada pelo diagrama de taxas apresentado na Figura 1, na qual $n$ é o número de clientes no sistema:

Figura 1 - Diagrama de taxa da fila M/M/1
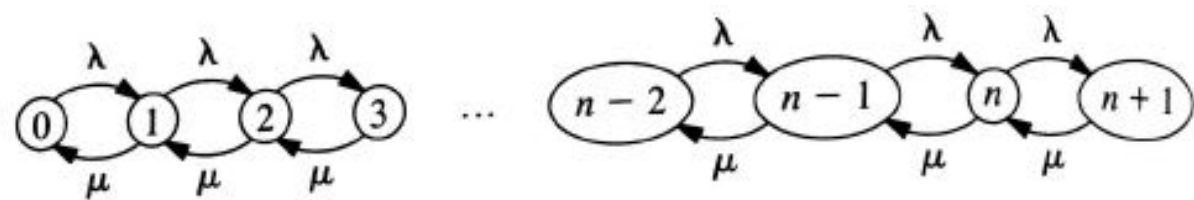

Fonte: Hillier e Lieberman (2013)

A taxa de utilização indica a estabilidade do sistema permitindo o estudo analítico do sistema de filas conforme Andrade (2002). A taxa de utilização e os parâmetros operacionais podem ser calculados para o estudo analítico destes sistemas por meio das seguintes equações apresentadas na Tabela 1. 
Tabela 1 - Parâmetros Operacionais do modelo M/M/1, $\lambda<\mu$.

\begin{tabular}{lccc}
\hline Parâmetros & Símbolo & Fórmula & \\
\hline Número esperado do sistema & $L$ & $\frac{\lambda}{(\mu-\lambda)}$ & (2) \\
Número esperado na fila & $L_{q}$ & $\frac{\lambda^{2}}{\mu(\mu-\lambda)}$ & (3) \\
$\begin{array}{l}\text { Tempo previsto de espera (inclui o tempo } \\
\text { de serviço) }\end{array}$ & $W$ & $\frac{1}{\mu-\lambda}$ & (4) \\
Tempo previsto na fila & $W_{q}$ & $\frac{\lambda}{\mu(\mu-\lambda)}$ & (5) \\
Probabilidade que o sistema esteja vazio & $P_{0}$ & $1-\frac{\lambda}{\mu}$ & (6) \\
\hline
\end{tabular}

Fonte: Adaptado de Moore \& Wheatherford (2005) apud Barbosa et al. (2009)

O sistema de filas com mais de um servidor (M/M/s), assim como no sistema de fila $M / M / 1$, processa as chegadas e 0 atendimento conforme a distribuição de Poisson e a distribuição exponencial negativa, respectivamente, porém o número de servidores no sistema é indicado pela letra " $s$ " e os clientes são atendidos por ordem de chegada. A fila M/M/s pode ser representado pelo diagrama de taxas apresentado por Hillier e Lieberman (2013), na Figura 2.

Figura 2 - Diagrama de taxa da fila M/M/s
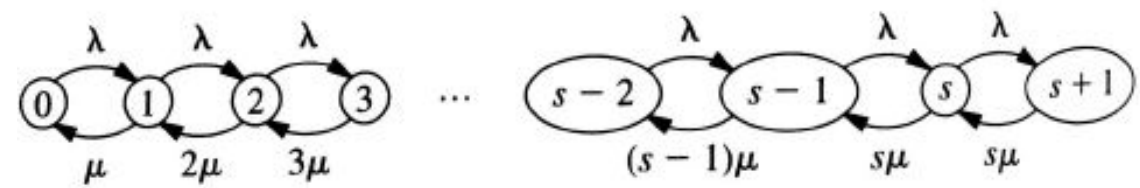

Fonte: Hillier e Lieberman (2013)

A taxa de utilização do sistema de filas M/M/s é calculada pela Equação 7 :

$$
\rho=\frac{\lambda}{s \mu}
$$

E as equações para o cálculo dos parâmetros operacionais para o sistema de filas M/M/s estão apresentados na Tabela 2. 
Tabela 2 - Parâmetros operacionais do modelo M/M/s, $\lambda<s \mu$

\begin{tabular}{lccr}
\hline Parâmetros & Símbolo & Fórmula \\
\hline $\begin{array}{l}\text { Probabilidades de o sistema estar } \\
\text { vazio }\end{array}$ & $P_{0}$ & $\frac{1}{\left(\sum_{j=0}^{s-1} \frac{\rho^{j}}{j !}+\frac{\rho^{s}}{(s-1) !(s-\rho)}\right)}$ \\
$\begin{array}{l}\text { Probabilidades de que todos os canais } \\
\text { estejam ocupados quando } n \leq s\end{array}$ & $P_{\text {ocup }}$ & $\frac{\rho^{n}}{n !} P_{0}$ \\
$\begin{array}{l}\text { Probabilidades de que todos os canais } \\
\text { estejam ocupados quando } n>s\end{array}$ & $P_{\text {ocup }}$ & $\frac{\rho^{s}}{(s-1) !(s-\rho)} P_{0}$ \\
Número esperado na fila & $L_{q}$ & $\frac{P_{0}(\lambda / \mu)^{s} \rho}{s !(1-\rho)^{2}}$ \\
Número esperado no sistema & $L$ & $L_{q}+\rho$ \\
Tempo previsto na fila & $W_{q}$ & $\frac{L_{q}}{\lambda}$ \\
$\begin{array}{l}\text { Tempo previsto de espera (inclui o } \\
\text { tempo de serviço) }\end{array}$ & $W$ & $W_{q}+\frac{1}{\mu}$ \\
\hline
\end{tabular}

Fonte: Adaptado de Hillier \& Lieberman (2006) apud Barbosa et al. (2009)

\section{METODOLOGIA}

O artigo foi desenvolvido de acordo com a metodologia de Chwif \& Medina (2010), e está estruturado em três grandes etapas conforme estão descritas a seguir e podem ser observadas na figura 3.

Figura 3 - Metodologia de Simulação

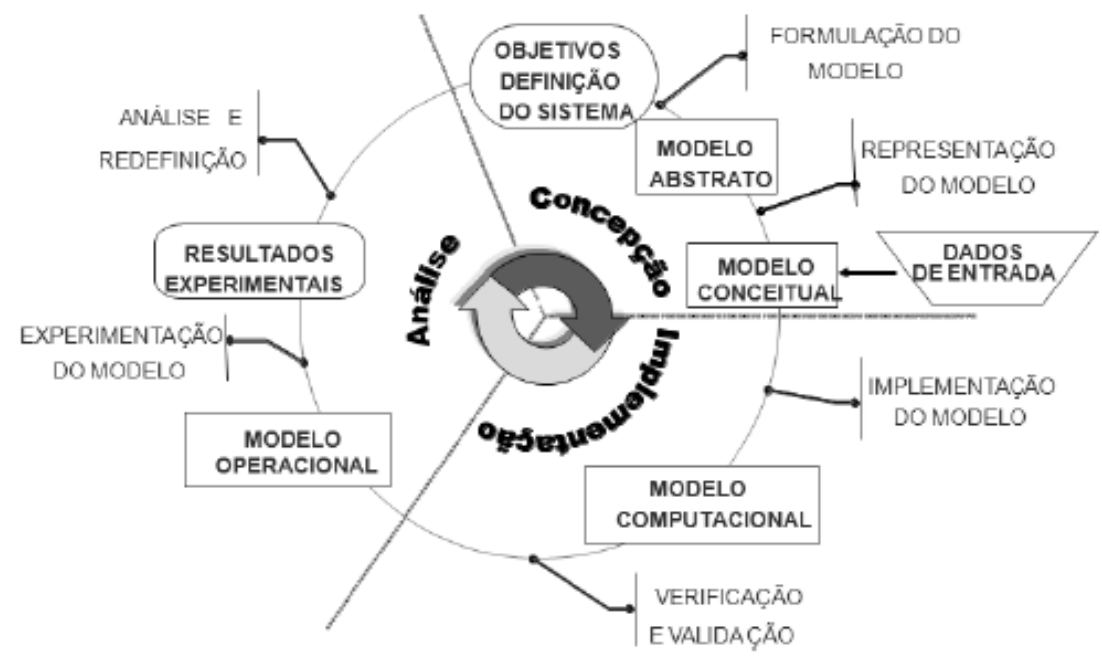

Fonte: Chwif (1999) apud Chwif e Medina (2010)

Revista Produção Online, Florianópolis, SC, v. 17, n. 1, p. 49-75, jan./mar. 2017. 
a) Concepção ou formulação do modelo. Esta etapa consiste inicialmente no entendimento claro do sistema a ser simulado e de seus objetivos. Em seguida deve ser estipulado o escopo do modelo, suas hipóteses e o nível de detalhamento. Após estas definições estabelecidas, ainda nesta etapa, é realizada a coleta de dados de forma a garantir a coleta correta dos dados para não prejudicar o desempenho do modelo. Por fim, o modelo deve ser representado de acordo com técnicas de representação de modelos de simulação para elaborá-lo na forma de um modelo conceitual;

Nesta etapa do estudo foi realizado o entendimento dos sistemas de filas M/M/1 e M/M/2 e suas representações. Também foram definidas as fórmulas conceituais que iriam ser utilizadas e os processos dos dados de entrada do sistema (processo de Poisson para o processo de chegada e a distribuição exponencial para os tempos de atendimento);

b) Implementação do modelo. $\mathrm{Na}$ fase de implementação, o modelo computacional é desenvolvido com base no modelo conceitual por meio da utilização de um software de simulação comercial. Em seguida o modelo computacional deve ser comparado com o modelo conceitual, a fim de validar a operação do sistema com o que foi estabelecido na etapa de concepção. Esta validação ocorre por meio da análise dos resultados do modelo computacional e comparados ao sistema real;

$\mathrm{Na}$ segunda etapa todas as fórmulas conceituais para modelar o comportamento dinâmico do sistema foram adaptadas para o software Excel, devido à facilidade de manuseio e a simplicidade do sistema, e o resultado foi gerado. Este resultado foi comparado com o modelo conceitual e validado;

c) Análise dos resultados do modelo. Posteriormente à etapa de implementação, em que o modelo do software foi validado, esta etapa de análise consiste na realização dos experimentos de forma a originar o modelo experimental. Várias "rodadas" do modelo são realizadas e os resultados são documentados e, em caso de necessidade, o modelo computacional é modificado e as "rodadas" são reiniciadas. Por fim, os resultados são documentados e conclusões e recomendações podem ser realizadas. 
Na última etapa, após a validação de ambos os modelos considerando alguns valores para tempo de aquecimento, foram realizadas replicações e as médias destes resultados foram comparadas novamente ao modelo teórico e conclusões a respeito do sistema foram realizadas.

\section{EXPERIMENTOS}

Para representação dos conceitos apresentados foram realizados alguns experimentos, por meio de uma simulação de filas na ferramenta Excel, de um servidor único e de dois servidores, com a disciplina da fila do tipo first in, first out (FIFO) e comparando-os com os resultados do modelo teórico de fila M/M/1 e M/M/2. Para esta simulação foi utilizado o sistema Microsoft Excel devido a simplicidade do sistema a ser simulado e facilidade de utilização frente aos softwares de simulação.

As fórmulas do modelo teórico com as quais serão comparados aos resultados da simulação estão apresentadas nas Equações de número 2, 3, 4, 5, e 6.

Para o desenvolvimento da simulação das filas M/M/1 no software Excel foram adotadas as Equações de 17 a 22, propostas por Perin (1995) e adaptadas para os experimentos:

$$
\begin{gathered}
r_{i}=\frac{-\ln (1-u)}{\lambda} \\
s_{i}=\frac{-\ln (1-u)}{\mu} \\
a_{i}=a_{i-1}+r_{i} \\
b_{i}=\max \left(a_{i} ; c_{i-1}\right) \\
c_{i}=b_{i}+s_{i} \\
w_{i}=b_{i}-a_{i} \\
u_{i}=c_{i}-a_{i} \\
o_{i}=b_{i}-c_{i-1}
\end{gathered}
$$

Em que:

$r_{i}$ - Tempo entre as chegadas (TEC);

Si - Tempo de atendimento;

$a_{i}$ - Instante de chegada;

$b_{i}$ - Início de atendimento;

$C_{i}-$ Instante do fim do atendimento;

$W_{i}$ - Tempo de espera na fila;

Revista Produção Online, Florianópolis, SC, v. 17, n. 1, p. 49-75, jan./mar. 2017. 
$u_{i}$ - Tempo do sistema;

oi-Tempo ocioso;

$u$ - Número aleatório ente 0 e 1.

Para a realização da simulação manual no Excel do modelo de servidor único e comparação com o modelo teórico, foram adotados os valores de entrada: tempo médio de chegada (TEC), $E\left(X_{1}\right)$, de 1 minuto; e o tempo médio de atendimento $E\left(X_{2}\right)$, de 0,5 minutos, modelados como variáveis aleatórias com distribuição exponencial. Desta forma, os valores da chegada por unidade de tempo $(\lambda)$ e atendimento por unidade de tempo $(\mu)$, podem ser encontrados através da inversa de $E\left(X_{1}\right)$ e $E\left(X_{2}\right)$ conforme as fórmulas 23 e 24 :

$$
\begin{aligned}
& \lambda=\frac{1}{E\left(X_{1}\right)} \\
& \mu=\frac{1}{\left(E X_{2}\right)}
\end{aligned}
$$

Em que:

$\lambda$ - Taxa de chegada;

$\mu$ - Nível de serviço;

$\mathrm{E}\left(X_{1}\right)$ - Tempo médio entre as chegadas;

$\mathrm{E}\left(\mathrm{X}_{2}\right)$ - Tempo médio de atendimento.

Sendo assim, tem-se que o valor da taxa de chegada $\lambda$ é 1 e o nível de serviço $\mu$ é 2 .

Na simulação no Excel os valores de $r_{\mathrm{i}}$ e $s_{\mathrm{i}}$ foram gerados através do método da transformada inversa, de forma aleatória, conforme pode ser observado na Tabela 3 , e aplicados para as $n=1.000$ chegadas de usuários, sendo " $n$ " o número de usuários no sistema. A Tabela 3 também apresenta as Equações 1 e de 15 a 24 apresentadas acima. 
Tabela 3 - Planilha de simulação da fila M/M/1

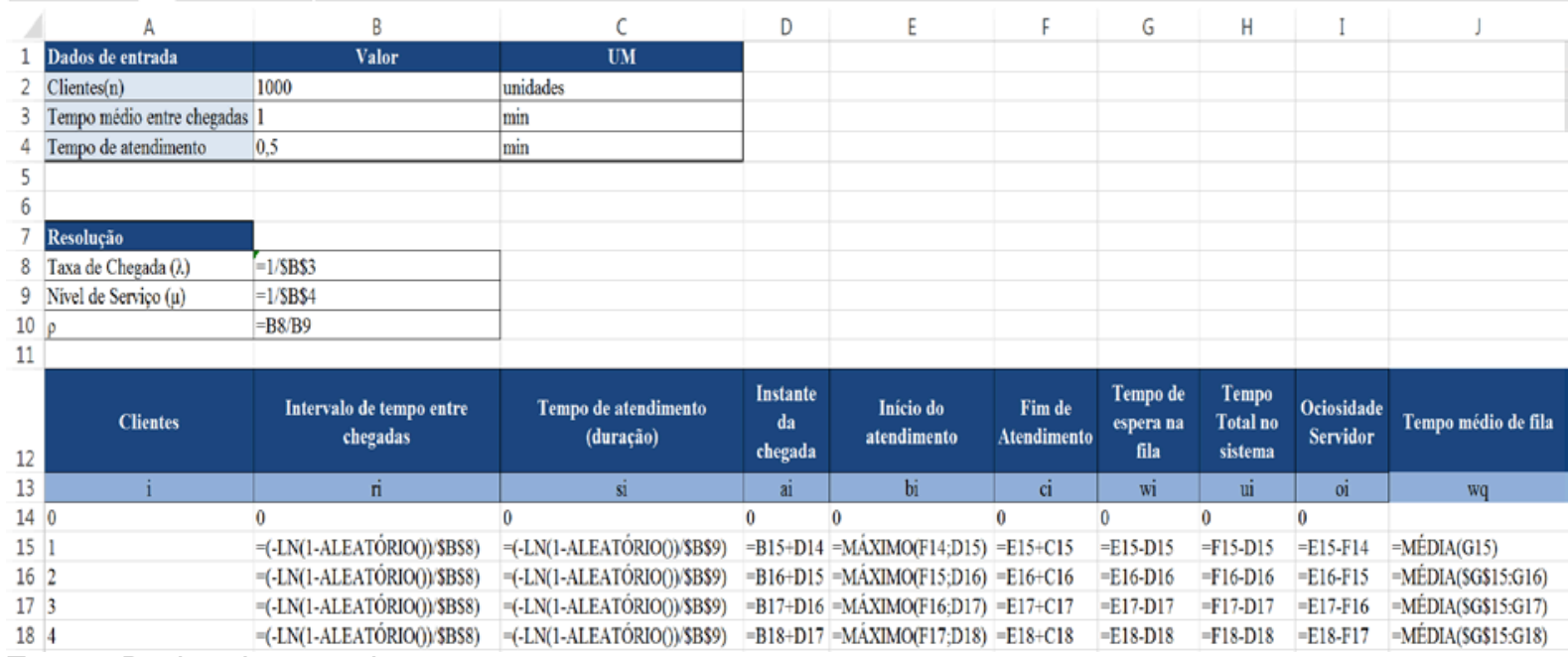

Fonte: Dados da pesquisa

Após a geração dos valores, como pode ser observado na Tabela 4, foram desenvolvidos dois histogramas para avaliar se os valores gerados pela simulação dos tempos entre chegadas e os tempos de atendimento se aproximam dos valores encontrados no modelo exponencial teórico. O Gráfico 1 apresenta o histograma dos tempos entre chegadas e o Gráfico 2 apresenta o histograma dos tempos de atendimentos.

Tabela 4 - Valores encontrados da planilha de simulação da fila M/M/1

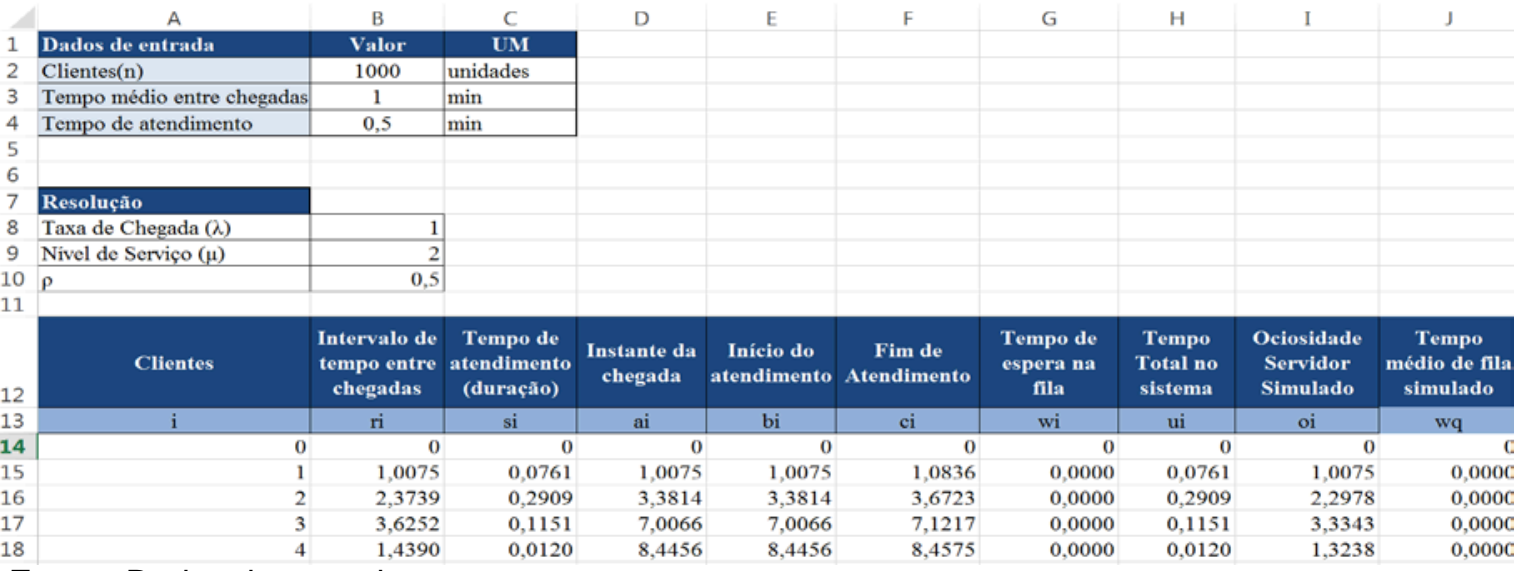

Fonte: Dados da pesquisa 
Gráfico 1 - Tempo entre as chegadas $\left(r_{1}\right)$

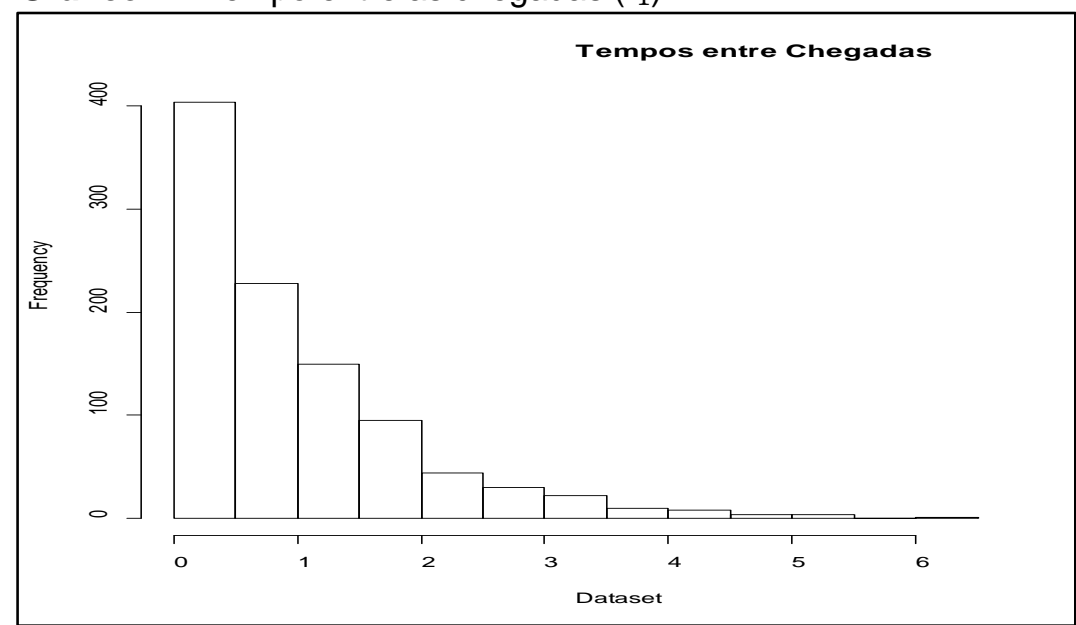

Fonte: Dados da pesquisa

O Gráfico 1 foi gerado a partir dos dados de $r_{i}$, e apresentou uma média de 0,9752 e variância de 0,9168, sendo estes resultados próximos ao modelo teórico, cujos valores da média e variância encontrados a partir da aplicação das fórmulas média $=1 / \lambda$ e variância $=1 / \lambda^{2}$, são iguais a 1 .

O Gráfico 2 gerado a partir dos dados de si, também apresentaram valores próximos aos teóricos. O valor simulado de si médio foi de 0,4947 e variância de 0,2329 sendo o valor teórico da média de 0,5 e a variância teórica de 0,25 , encontrado pela fórmula média $=1 / \mu$ e variância $=1 / \mu^{2}$.

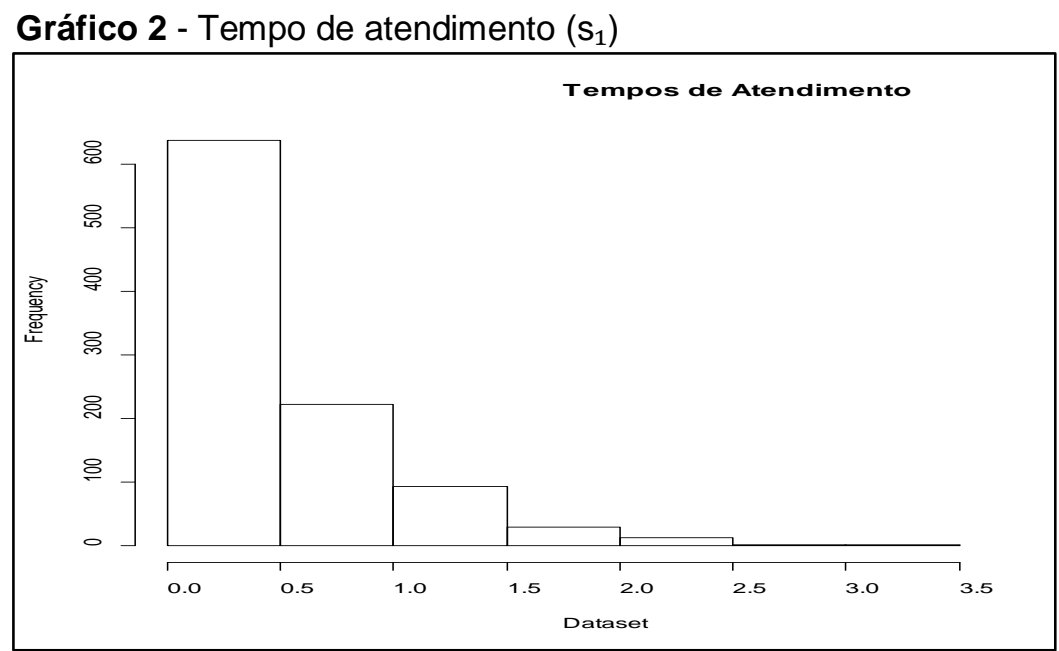

Fonte: Dados da pesquisa

Em seguida foram analisadas as medidas do tempo médio de espera na fila $(W q)$ e a ocupação do servidor ( $\rho$ ) para os 1.000 clientes no sistema. Como se pode observar no Gráfico 3 do tempo de médio de fila, e no Gráfico 4 da ocupação do Revista Produção Online, Florianópolis, SC, v. 17, n. 1, p. 49-75, jan./mar. 2017. 
servidor, a escolha do valor arbitrário de 1.000 usuários no sistema, foi suficiente para convergir o resultado simulado ao teórico de $W q=0,5$ e $\rho=0,5$, respectivamente, e para alcançar o regime estável.

Gráfico 3 - Wq simulado x teórico para fila M/M/1

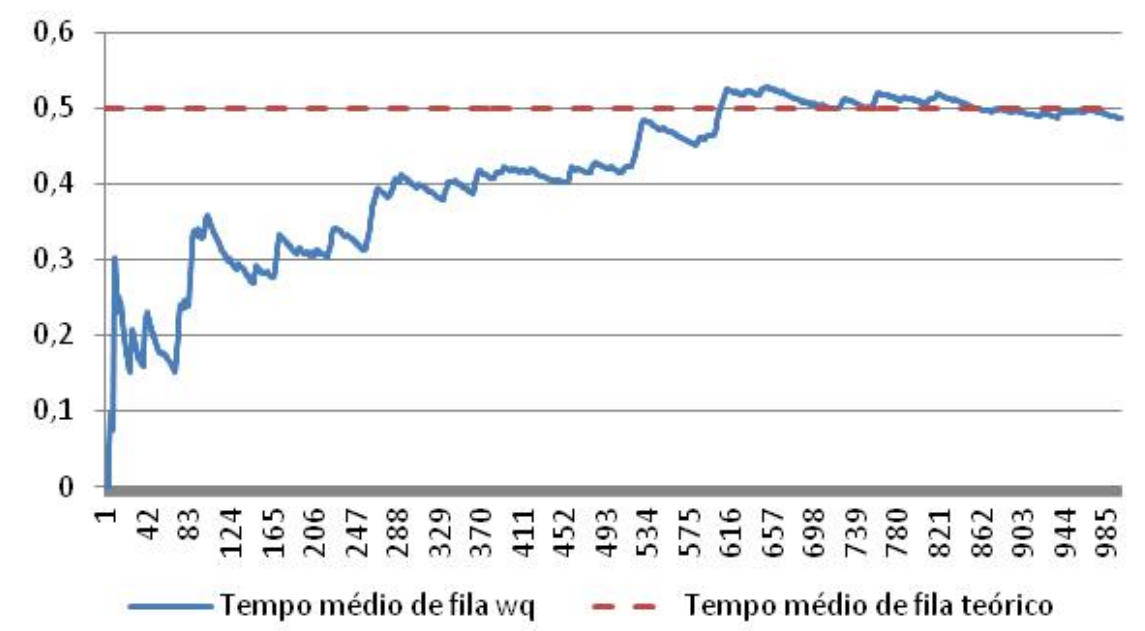

Fonte: Dados da pesquisa

Gráfico 4 - $\rho$ simulado x teórico para fila M/M/1

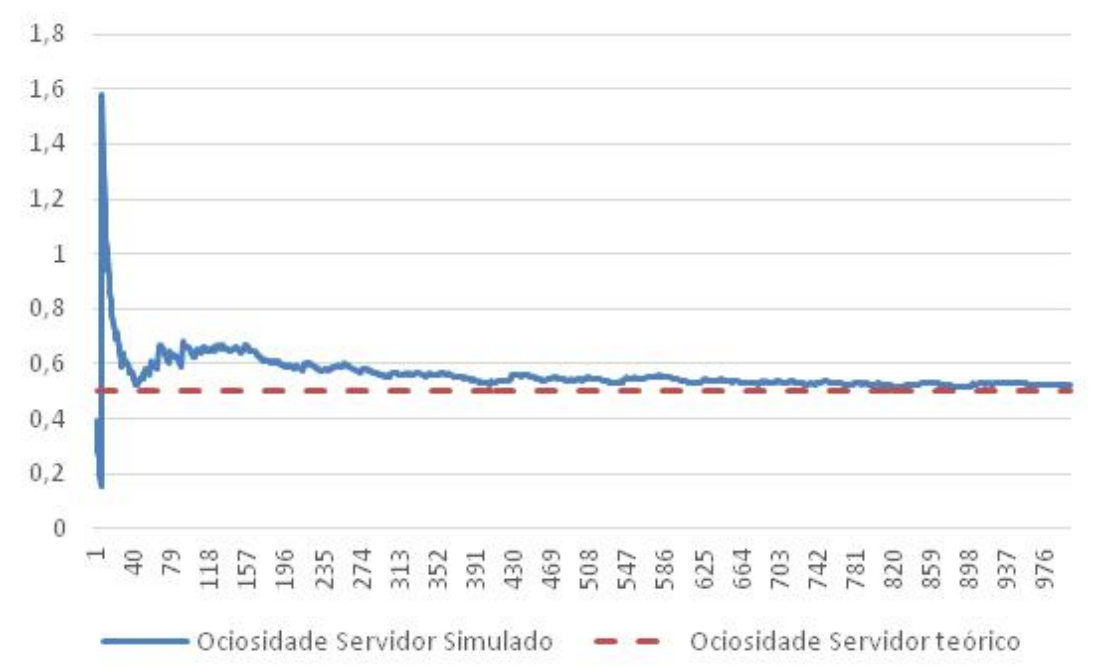

Fonte: Dados da pesquisa

No entanto para ter uma melhor análise da simulação, esta foi repetida 10 vezes e os resultados, encontrados pela aplicação das equações 25 e 26, foram coletados para minimizar a variabilidade do sistema conforme apresentado nas Tabelas 5 e 6 . Ainda nas Tabelas 5 e 6 para finalizar as análises da fila M/M/1, o tempo médio entre as chegadas foi reduzido de 0,$9 ; 0,8 ; 0,7 ; 0,6$ até 0,505 e replicados 10 
vezes para avaliar o comportamento do sistema com a redução dos intervalos de chegadas.

$$
\begin{gathered}
W q=\frac{\sum w_{i}}{n} \\
\rho=1-\left(\frac{\sum o_{i}}{c_{1000}}\right)
\end{gathered}
$$

Em que:

$W q$ - Tempo médio na fila;

$w_{i}-$ Tempo de espera na fila;

$n$ - Número de servidores;

$\rho$ - Taxa de ocupação;

$o_{\mathrm{i}}$ - Tempo ocioso;

$C_{1000}$ - Fim do Atendimento do cliente número 1.000

\begin{tabular}{|c|c|c|c|c|c|c|c|c|c|c|c|c|c|c|c|}
\hline \multirow{2}{*}{$\begin{array}{l}\text { Tempo entre } \\
\text { chegadas }\end{array}$} & \multirow{2}{*}{$\lambda$} & \multirow{2}{*}{$\mu$} & \multicolumn{10}{|c|}{ Replicaçōes } & \multirow{2}{*}{ w médio } & \multirow{2}{*}{ Variância } & \multirow{2}{*}{ Wa teóricc } \\
\hline & & & 1 & 2 & 3 & 4 & 5 & 6 & 7 & 8 & 9 & 10 & & & \\
\hline 1 & 1 & 2 & 0,4155 & 0,5172 & 0,4867 & 0,3966 & 0,5545 & 0,5615 & 0,4230 & 0,3638 & 0,4585 & 0,4040 & 0,4581 & 0,0048 & 0,5000 \\
\hline 0,9 & 1,1111 & 2 & 0,5586 & 0,8008 & 0,5523 & 0,7180 & 0,4923 & 0,4590 & 0,6066 & 0,6536 & 0,0023 & 0,6466 & 0,6370 & 0,0144 & 0,6250 \\
\hline 0,8 & 1,25 & 2 & 0,7359 & 0,9673 & 1,4800 & 0,7118 & 0,7280 & 0,7277 & 1,2283 & 0,7947 & 1,1283 & 0,8403 & 0,9342 & 0,0655 & 0,8333 \\
\hline 0,7 & 1,4286 & 2 & 1,1486 & 1,0886 & 0,9405 & 2,1697 & 0,9952 & 1,1789 & 1,0914 & 1,3442 & 1,1285 & 1,0359 & 1,2122 & 0,1254 & 1,2501 \\
\hline 0,6 & 1,6666 & 2 & 1,9807 & 2,1674 & 3,1723 & 4,0505 & 1,4993 & 1,9123 & 1,6153 & 3,9232 & 1,0066 & 2,2004 & 2,4136 & 0,9144 & 2,4994 \\
\hline 0,505 & 1,98 & 2 & 10,1418 & 10,7264 & 18,4012 & 9,6592 & 23,1944 & 18,9120 & 33,0478 & 7,8025 & 5,5634 & 19,4243 & 15,6073 & 71,7597 & 49,5000 \\
\hline
\end{tabular}

Tabela 5 - Tempo médio de espera de fila e replicações

Fonte: Dados da pesquisa

\begin{tabular}{|c|c|c|c|c|c|c|c|c|c|c|c|c|c|c|c|}
\hline \multirow{2}{*}{$\begin{array}{l}\text { Tempo entre } \\
\text { chegadas }\end{array}$} & \multirow{2}{*}{$\lambda$} & \multirow[b]{2}{*}{$\mu$} & \multicolumn{10}{|c|}{ Replicaçōes } & \multirow[b]{2}{*}{ p médio } & \multirow[b]{2}{*}{ Variânica } & \multirow[b]{2}{*}{ p teórico } \\
\hline & & & 1 & 2 & 3 & 4 & 5 & 6 & 7 & 8 & 9 & 10 & & & \\
\hline 1 & 1 & 2 & $48 \%$ & $55 \%$ & $53 \%$ & $48 \%$ & $55 \%$ & $50 \%$ & $48 \%$ & $45 \%$ & $40 \%$ & $49 \%$ & $50 \%$ & 0,0011 & 0,5000 \\
\hline 0,9 & 1,1111 & 2 & $53 \%$ & $59 \%$ & $55 \%$ & $59 \%$ & $51 \%$ & $54 \%$ & $55 \%$ & $54 \%$ & $50 \%$ & $57 \%$ & $56 \%$ & 0,0007 & 0,5555 \\
\hline 0,8 & 1,25 & 2 & $65 \%$ & $66 \%$ & $64 \%$ & $65 \%$ & $61 \%$ & $62 \%$ & $63 \%$ & $61 \%$ & $65 \%$ & $64 \%$ & $64 \%$ & 0,0004 & 0,6250 \\
\hline 0,7 & 1,4286 & 2 & $75 \%$ & $70 \%$ & $70 \%$ & $77 \%$ & $66 \%$ & $66 \%$ & $70 \%$ & $72 \%$ & $69 \%$ & $69 \%$ & $71 \%$ & 0,0013 & 0,7142 \\
\hline 0,6 & 1,6666 & 2 & $81 \%$ & $83 \%$ & $90 \%$ & $89 \%$ & $78 \%$ & $83 \%$ & $74 \%$ & $84 \%$ & $79 \%$ & $83 \%$ & $82 \%$ & 0,0023 & 0,8333 \\
\hline 0,505 & 1,98 & 2 & $99 \%$ & $99 \%$ & 100\% & $95 \%$ & $99 \%$ & $96 \%$ & $98 \%$ & $98 \%$ & $97 \%$ & $100 \%$ & $98 \%$ & 0,0003 & 0,9901 \\
\hline
\end{tabular}

Tabela 6 - Ocupação do servidor

Fonte: Dados da pesquisa

Ao observar a Tabela 5 verifica-se que as 10 replicações são necessárias para evitar erros na análise do sistema, caso analisados sem a média. Ao se analisar a Revista Produção Online, Florianópolis, SC, v. 17, n. 1, p. 49-75, jan./mar. 2017. 
variabilidade dos resultados do tempo médio de espera de fila dos valores de entrada $\lambda=1$ e $\mu=2$, pode-se constatar um coeficiente de variação de 15,1\%, em relação ao valor do $w$ médio. O coeficiente de variação é calculado dividindo-se o valor do desvio padrão (raiz quadrada da variância) pelo valor médio e em seguida multiplicando o resultado por 100. Desta forma utilizando a média das 10 replicações os valores dos resultados ficam mais confiáveis. Na replicação 8, por exemplo, o valor obtido foi de 0,3638 e na replicação 6 , foi de 0,5616. Caso apenas uma destas replicações tivesse sido utilizada os erros seriam significativos (frente ao valor teórico, que é de 0,5000).

Ainda analisando as Tabelas 5 e 6 percebe-se que à medida que o tempo médio entre as chegadas ficam menores (aceleração da taxa de chegada), o tempo médio de espera na fila aumenta de forma expressiva, de 0,5 min para 49,5 min, segundo o modelo teórico. Observa-se, com exceção do TEC de 0,505 min, que os resultados da simulação ( $w$ médio) ficaram bem próximos ao modelo teórico. A taxa de ocupação do servidor também aumenta de forma significativa com um aumento do valor de $50 \%$ de ocupação para uma média simulada de 98\%.

Entretanto, quando o intervalo entre as chegadas diminuiu para 0,505 min, os valores encontrados na simulação do tempo de espera na fila apresentaram uma diferença inaceitável em relação ao valor teórico, pois o tempo médio de espera na fila simulado foi de 15,6873 min quando era esperado um valor de $W q$ teórico de 49,5 min. Desta forma, diante da diferença entre o valor simulado e teórico é necessário aumentar o número de clientes chegando no sistema até que seja atingido um regime estável e os resultados apresentem uma diferença mínima, como nas simulações para outros valores de TEC.

Sendo assim foram realizadas novas simulações aumentando o número de clientes para 2.000; 5.000; 10.000; 20.000; 50.000; 70.000; 80.000; 90.000 e 100.000, a fim de avaliar se os valores simulados atingem um regime estável e se aproximam dos valores teóricos. Os resultados obtidos foram apresentados na Tabela 7. Pode-se observar que a partir de $n=90.000$, os resultados simulados se aproximam dos resultados teóricos. 
Tabela 7 - Comparação entre tempos médios de filas

\begin{tabular}{|c|c|c|c|c|c|c|c|c|c|c|c|c|c|c|c|c|}
\hline \multirow{2}{*}{$\begin{array}{l}\text { Tempo entre } \\
\text { chegadas } \\
\end{array}$} & \multirow[b]{2}{*}{$\lambda$} & \multirow[b]{2}{*}{$\mu$} & \multirow[b]{2}{*}{$n$} & \multicolumn{10}{|c|}{ Replicaçōes } & \multirow{2}{*}{ w médic } & \multirow[b]{2}{*}{ Variância } & \multirow[b]{2}{*}{ Wq téórico } \\
\hline & & & & 1 & 2 & 3 & 4 & 5 & 6 & 7 & 8 & 9 & 10 & & & \\
\hline 0,505 & 1,98 & 2 & 1.000 & 11,7892 & 20,5868 & 12,4124 & 10,7073 & 11,0672 & 27,6358 & 17,9666 & 13,2947 & 13,4753 & 19,8756 & 15,8011 & 30,2018 & 49,5000 \\
\hline 0,505 & 1,98 & 2 & 2.000 & 18,6612 & 14,0941 & 23,3137 & 17,7777 & 18,8180 & 29,8564 & 20,3938 & 18,9715 & 25,7460 & 18,1353 & 20,5769 & 20,6128 & 49,5000 \\
\hline 0,505 & 1,98 & 2 & 5.000 & 30,7495 & 21,4511 & 19,8783 & 24,0544 & 36,9561 & 30,4412 & 26,8302 & 22,2275 & 37,1038 & 40,3133 & 29,0006 & 53,0361 & 49,5000 \\
\hline 0,505 & 1,98 & 2 & 10.000 & 30,3789 & 31,1780 & 43,8987 & 45,7887 & 24,2968 & 27,7876 & 31,8630 & 27,0214 & 26,5662 & 28,4517 & 31,0052 & 52,3437 & 49,5000 \\
\hline 0,505 & 1,98 & 2 & 20.000 & 49,1075 & 36,8232 & 29,2185 & 32,6510 & 36,0838 & 44,4242 & 37,1290 & 39,0214 & 47,1373 & 31,3993 & 38,2995 & 44,0217 & 49,5000 \\
\hline 0,505 & 1,98 & 2 & 50.000 & 42,7509 & 36,7942 & 37,4833 & 38,3740 & 42,7028 & 47,3183 & 35,5112 & 41,2357 & 47,2055 & 49,5610 & 41,8937 & 24,0926 & 49,5000 \\
\hline 0,505 & 1,98 & 2 & 70.000 & 41,5908 & 40,7045 & 47,4839 & 41,6533 & 47,6446 & 41,1409 & 48,6467 & 42,9208 & 47,7193 & 45,5493 & 44,5054 & 10,2447 & 49,5000 \\
\hline 0,005 & 1,98 & 2 & 80.000 & 51,6129 & 51,1628 & 47,3586 & 52,1957 & 44,3336 & 44,5962 & 44,3396 & 50,0205 & 48,3974 & 51,7343 & 48,6552 & 10,0071 & 49,5000 \\
\hline 0,505 & 1,98 & 2 & 90.000 & 47,6593 & 49,1498 & 52,8357 & 51,9003 & 49,9128 & 51,3240 & 45,5042 & 49,5191 & 53,5876 & 44,8536 & 49,6246 & 8,6673 & 49,5000 \\
\hline 0,505 & 1,98 & 2 & 100.000 & 48,5784 & 50,2843 & 52,1356 & 46,9427 & 47,0691 & 45,6349 & 53,5876 & 49,7948 & 49,0610 & 53,4848 & 49,6573 & 7,6020 & 49,5000 \\
\hline
\end{tabular}

Fonte: Dados da pesquisa

Como se pode demonstrar na Tabela 7, à medida que o número de clientes no sistema aumentou, o tempo médio de fila atingiu o regime estável, reduzindo a variabilidade, e se aproximou cada vez mais dos valores teóricos. Portanto, fica evidente a necessidade do aumento da quantidade de clientes no sistema, da análise do tempo de aquecimento e das diversas replicações do modelo para se obter dados de saída confiáveis.

Para a simulação da fila $\mathrm{M} / \mathrm{M} / 2$, tanto no modelo teórico quanto no Excel, o número de servidores foi alterado para dois e os valores de entrada foram o TEC de 0,505 minutos, e o tempo médio de atendimento que permaneceu 0,5 minutos, sendo modelados também como variáveis aleatórias com distribuição exponencial para poder comparar os resultados com a fila M/M/1.

$\mathrm{Na}$ fila $\mathrm{M} / \mathrm{M} / 2$, desenvolvida no software Excel, os valores encontrados pelas aplicações das fórmulas 15, 16, 17, 19, 20, 21, 27, 28, 29 e 30, apresentados pelas Tabelas 8A e 8B, podem ser observados na Tabela 9.

$$
\begin{gathered}
b_{i}=\max \left\{a_{i}, \min \left\{c_{i-1}^{1}, c_{i-1}^{2}\right\}\right\} \\
c_{i}^{1}\left\{\begin{array}{c}
c_{i}, \text { se } c_{i-1}^{1} \leq c_{i-1}^{2} \\
c_{i-1}^{1}, \text { se } c_{i-1}^{1}>c_{i-1}^{2}
\end{array}\right\} \\
c_{i}^{2}\left\{\begin{array}{c}
c_{i-1}^{2}, \text { se } c_{i-1}^{1} \leq c_{i-1}^{2} \\
c_{i}, \text { se } c_{i-1}^{1}>c_{i-1}^{2}
\end{array}\right\} \\
o_{i}=\left\{\begin{array}{l}
b_{i}-c_{i-1}^{1}, \text { se } c_{i}=c_{i}^{1} \\
b_{i}-c_{i-1}^{2}, \text { se } c_{i} \neq c_{i}^{1}
\end{array}\right\}
\end{gathered}
$$



Em que:
$b_{i}$ - Início de atendimento;
$\mathrm{Ci}_{1}$ - Instante do fim do atendimento do servido 1 ;
$\mathrm{Ci}_{2}$ - Instante do fim do atendimento do servido 2
$o_{i}$ - Tempo ocioso da fila M/M/2.

Tabela 8A - Planilha de simulação da fila M/M/2

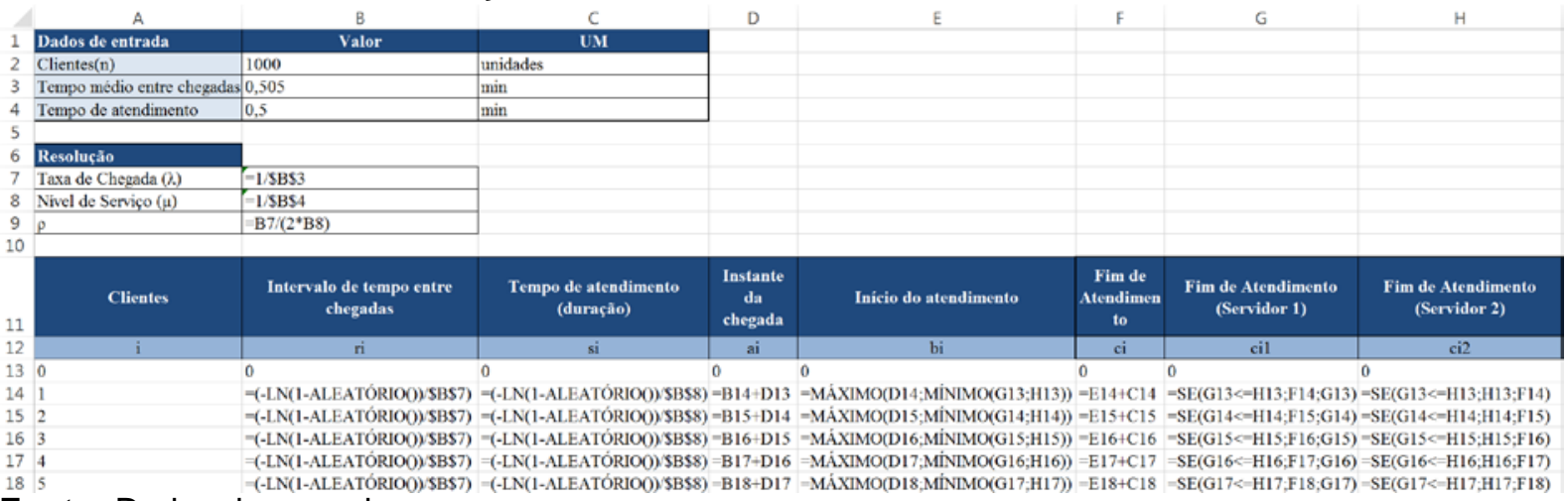

Fonte: Dados da pesquisa

Tabela 8B - Continuação da planilha de simulação da fila M/M/2

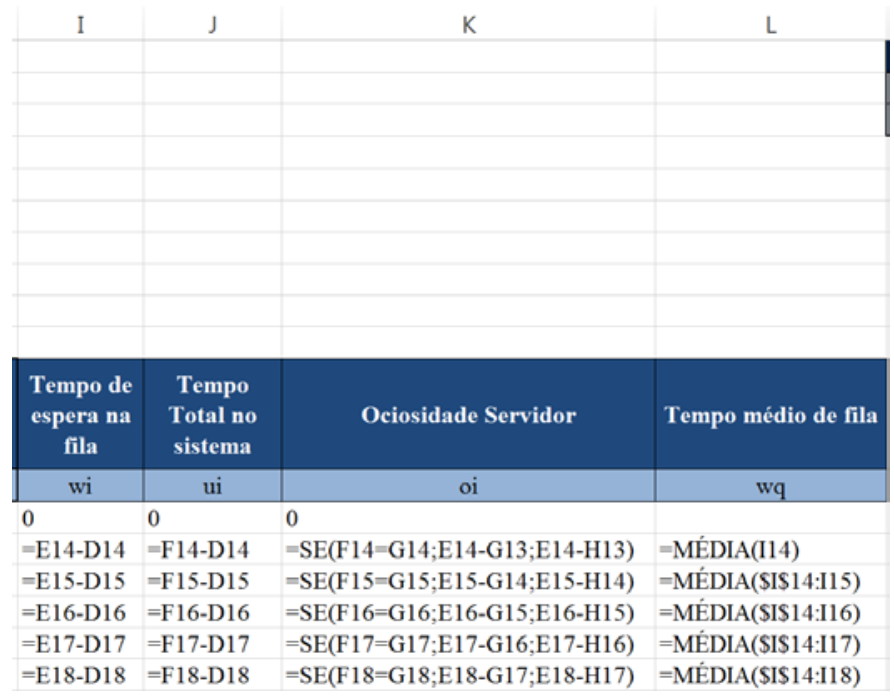

Fonte: Dados da pesquisa 
Tabela 9 - Valores encontrados da planilha de simulação da fila M/M/2

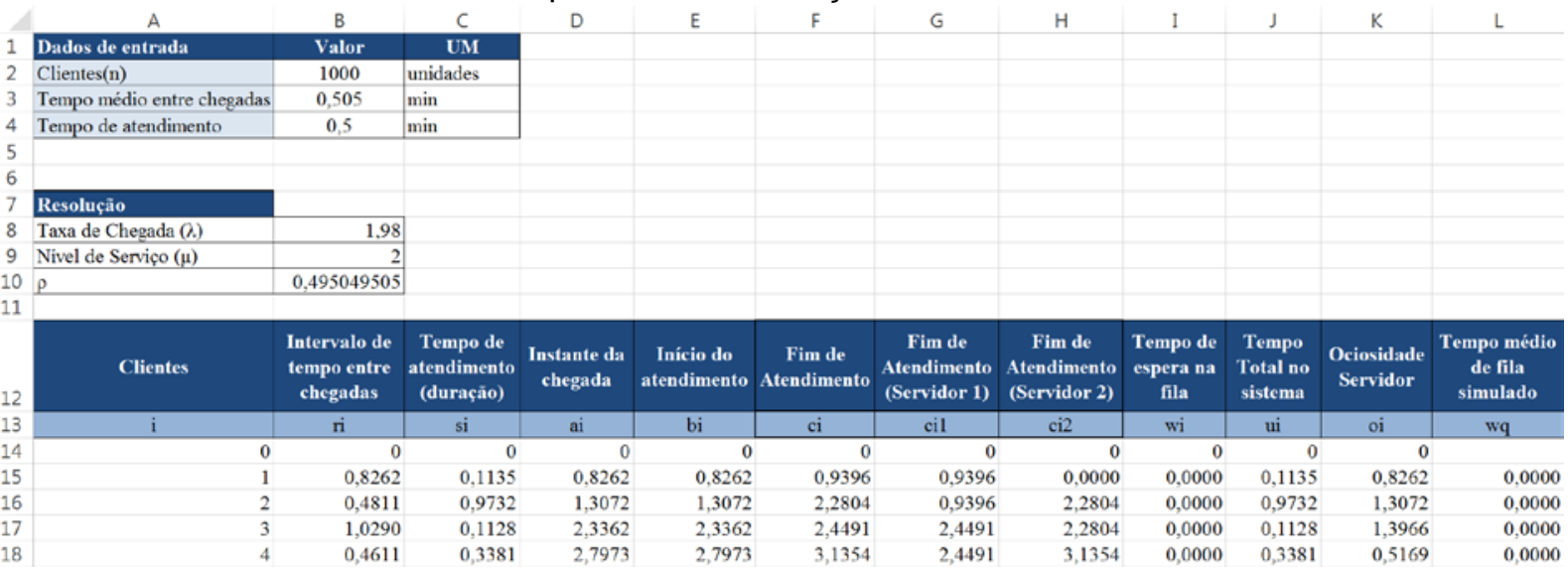

Fonte: Dados da pesquisa

Após a geração dos valores, os resultados apresentados pela simulação dos tempos entre chegadas e os tempos de atendimento também foram analisados pelo histograma, como pode ser observado nos Gráficos 5 e 6, a fim de avaliar se os resultados apresentados se aproximam dos valores do modelo exponencial teórico.

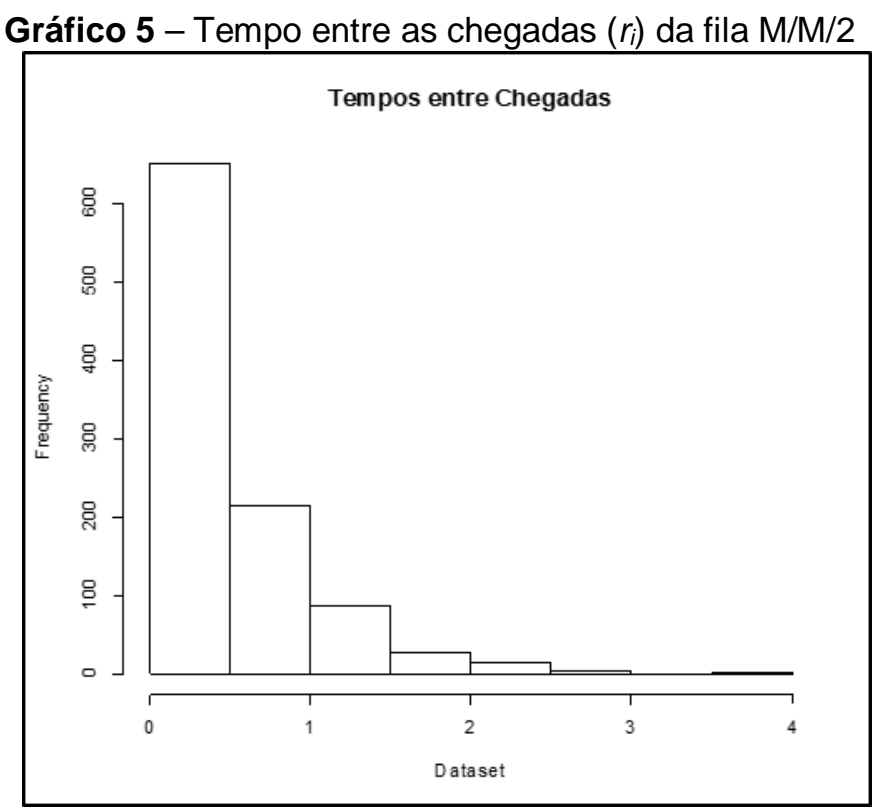

Fonte: Dados da pesquisa

O histograma do tempo entre as chegadas $\left(r_{i}\right)$ da fila M/M/2 apresentou uma média de 0,48 e variância de 0,24, valores estes próximos aos valores teóricos de 0,51 de média e 0,26 de variância. 


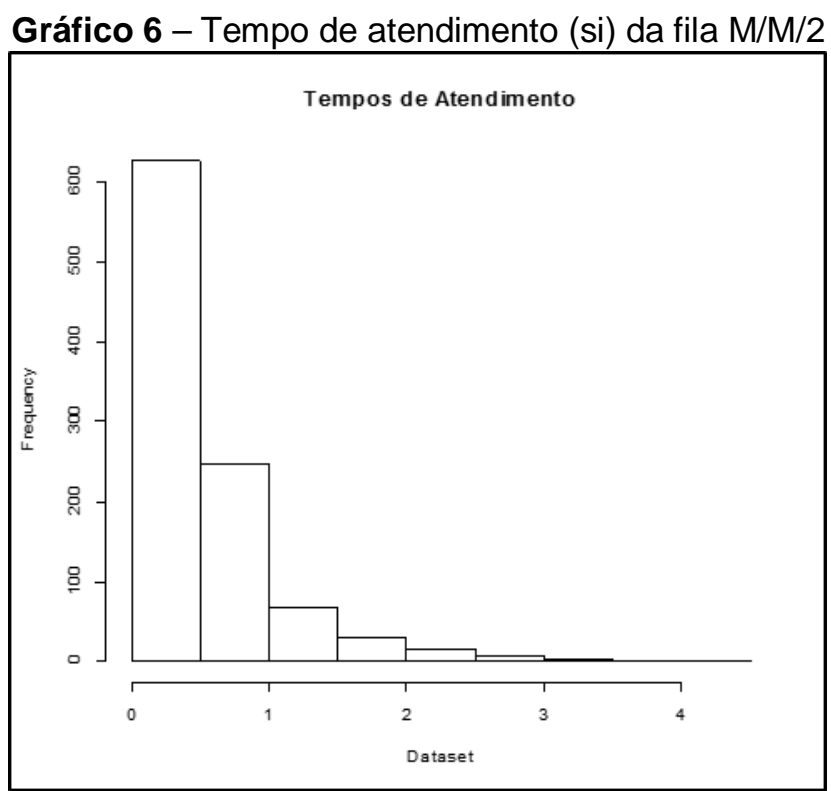

Fonte: Dados da pesquisa

Assim como o histograma dos tempos entre chegadas apresentado no Gráfico 5, o Gráfico 6 do histograma do tempo de atendimento (si) da fila M/M/2, apresentou o resultado próximo ao teórico, sendo os valores encontrados de 0,51 de média e 0,28 de variância e os valores teóricos de 0,50 de média e 0,25 de variância.

Ao analisar as medidas tempo médio de espera de fila e ocupação do servidor para os 1.000 usuários no sistema a fila M/M/2 obtidos pela aplicação das fórmulas 25 e 31, a fila M/M/2 apresentou um tempo médio de espera na fila (observado no Gráfico 7) acima do valor teórico, ou seja, convergiu para um valor acima do teórico e a ocupação do servidor abaixo, porém próximo ao valor teórico, alcançando o regime estável.

Resultados estes satisfatórios assim como os resultados do tempo médio de fila e ocupação do servidor da fila M/M/1.

$$
\rho=1-\left(\frac{\sum o_{i}}{2 \times c_{1000}}\right)
$$

Em que:

$\rho$ - Taxa de ocupação;

oi - Tempo ocioso;

$C_{1000}$ - Fim do Atendimento do cliente número 1.000. 
Gráfico 7 - Wq simulado x teórico para fila M/M/2

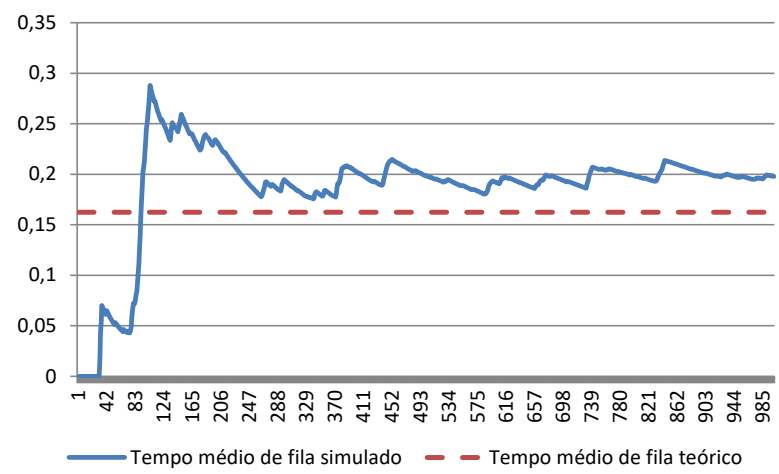

Fonte: Dados da pesquisa

Gráfico 8 - $\rho$ simulado $x$ teórico para fila $M / M / 2$

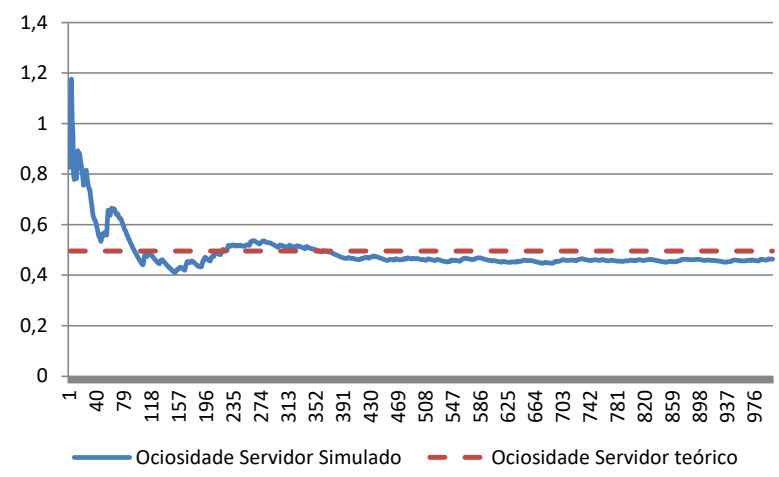

Fonte: Dados da pesquisa

Da mesma forma com que foi realizado na fila $M / M / 1$, para obter uma análise da simulação mais confiável, a simulação foi repetida 10 vezes de forma a obter um valor médio das simulações e compará-la aos valores teóricos, como pode ser observada na Tabela 10, e possibilitar uma comparação aos valores teóricos da fila $\mathrm{M} / \mathrm{M} / 1$.

Tabela 10 - Ocupação do servidor

\begin{tabular}{|c|c|c|c|c|c|c|c|c|c|c|c|c|c|c|c|c|}
\hline \multirow{2}{*}{$\begin{array}{c}\text { Tempo entre } \\
\text { chegadas }\end{array}$} & \multirow{2}{*}{$\lambda$} & \multirow{2}{*}{$\mu$} & \multirow{2}{*}{ Parâmetro } & \multicolumn{10}{|c|}{ Replicações } & \multirow{2}{*}{ Média } & \multirow{2}{*}{ Variância } & \multirow{2}{*}{$\begin{array}{l}\text { Valor } \\
\text { teórico }\end{array}$} \\
\hline & & & & 1 & 2 & 3 & 4 & 5 & 6 & 7 & 8 & 9 & 10 & & & \\
\hline \multirow{2}{*}{0,505} & \multirow{2}{*}{1} & & $W q$ & 0,1274 & 0,1844 & 0,1996 & 0,1773 & 0,1520 & 0,1526 & 0,1132 & 0,1703 & 0,1608 & 0,1123 & 0,1550 & 0,0009 & 0,1623 \\
\hline & & & 0 & $49 \%$ & $52 \%$ & $50 \%$ & $53 \%$ & $47 \%$ & $48 \%$ & $50 \%$ & $51 \%$ & $48 \%$ & $47 \%$ & $49 \%$ & 0,0004 & 0,4950 \\
\hline
\end{tabular}

Fonte: Dados da pesquisa

Conforme pode ser observado na Tabela 10, realizar as replicações são de extrema importância uma vez que os resultados das replicações do tempo médio de espera na fila $(W q)$ para 1.000 clientes no sistema variaram de 2\% a 29\% em relação ao tempo médio de espera na fila das 10 replicações de 0,1550 minutos, e apresentou 
um coeficiente de variação de 19,2\%. No entanto, apesar das replicações apresentarem um percentual alto do coeficiente de variação o resultado da média do tempo médio de espera na fila ficou próximo ao teórico.

Em relação ao percentual da ocupação dos servidores, estes apresentaram uma variação de 0\% a 7\% ao valor médio das 10 replicações de 49,48\%, e um coeficiente de variação de 3,95\%. Sendo o resultado da média das 10 replicações da ocupação do servidor de 49,48\% muito próximo do valor teórico de 49,50\%.

Logo percebe-se que, o tempo de espera de fila apesar de ter excedido o valor teórico, apresentado no Gráfico 7, atingiu o regime estável e reduziu de forma considerável quando comparado a fila M/M/1, reduzindo de 49,5 minutos para uma média de 0,1550 minutos como apresentado na Tabela 10.

E a taxa de ocupação do servidor, apresentada no Gráfico 8, apresentou um regime estável e melhores resultados, reduzindo de $98 \%$ da fila $\mathrm{M} / \mathrm{M} / 1$ para $49 \%$ na fila $\mathrm{M} / \mathrm{M} / 2$, permanecendo dentro dos limites máximos de ocupação de servidor e demonstrando que a escolha por colocar outro canal de atendimento é válida para estes valores de entradas no sistema de fila $\mathrm{M} / \mathrm{M} / 2$.

Ainda analisando a Tabela 10, apesar de apresentar resultados melhores para este sistema e apresentar os resultados médios mais próximos aos resultados teóricos, ainda é necessário aumentar a quantidade de clientes chegando no sistema até que os resultados médios do tempo médio de espera na fila e a ocupação do servidor apresentem uma diferença mínima em relação aos valores teóricos e um regime estável.

Dessa forma, novas simulações foram realizadas para a fila M/M/2, assim como foi realizado para a fila M/M/1, aumentando o número de clientes de 1.000 para 2.000; 5.000; 10.000; 20.000; 50.000; 70.000; 80.000; 90.000; 100.000, até que o tempo médio de espera de fila e a ocupação do servidor atingem um regime estável e apresentem uma diferença mínima em relação ao teórico, possibilitando uma comparação a fila M/M/1. Os resultados obtidos foram apresentados na Tabela 11. 
Tabela 11 - Comparação entre tempos médios de filas da fila M/M/2

\begin{tabular}{|c|c|c|c|c|c|c|c|c|c|c|c|c|c|c|c|c|}
\hline \multirow{2}{*}{$\begin{array}{l}\text { Tempo entre } \\
\text { chergatas }\end{array}$} & \multirow[b]{2}{*}{$\lambda$} & \multirow[b]{2}{*}{$\mu$} & \multirow[b]{2}{*}{$\mathrm{n}$} & \multicolumn{10}{|c|}{ Replicanoues } & \multirow[b]{2}{*}{ w medl } & \multirow[b]{2}{*}{ riana } & \multirow{2}{*}{ Wq teóric } \\
\hline & & & & 1 & 2 & 3 & 4 & 5 & 6 & 7 & 8 & 9 & 10 & & & \\
\hline 0,505 & 1,98 & 2 & 1.000 & 0,1274 & 0,1844 & 0,1996 & 0,1773 & 0,1520 & 0,1526 & 0,1132 & 0,1703 & 0,1608 & 0,1123 & 0,1550 & 0,0009 & 0,1623 \\
\hline 0,505 & 1,98 & 2 & 2.000 & 0,1585 & 0,1792 & 0,1880 & 0,1416 & 0,1543 & 0,1956 & 0,1672 & 0,1485 & 0,1528 & 0,1400 & 0,1626 & 0,0004 & 0,1623 \\
\hline 0,505 & 1,98 & & 5.000 & 0,1346 & 0,1529 & 0,1731 & 0,1323 & 0,1603 & 0,1954 & 0,1591 & 0,1499 & 0,1528 & 0,1496 & 0,1560 & 0,0003 & 0,1623 \\
\hline 0,505 & 1,98 & 2 & 10.000 & 0,1566 & 0,1480 & 0,1605 & 0,1658 & 0,1679 & 0,1768 & 0,1691 & 0,1595 & 0,1595 & 0,1520 & 0,1616 & 0,0001 & 0,1623 \\
\hline 0,505 & 1,98 & & 200000 & 0,1623 & 0,1569 & 0,1663 & 0,1555 & 0,1475 & 0,1594 & 0,1544 & 0,1681 & 0,1653 & 0,1717 & 0,1611 & 0,0001 & 0,1623 \\
\hline 0,505 & 1,98 & 2 & 50.000 & 0,1581 & 0,1539 & 0,6649 & 0,6558 & 0,1754 & 0,1629 & 0,1610 & 0,1695 & 0,1613 & 0,1695 & 0,1642 & 0,0000 & 0,1623 \\
\hline
\end{tabular}

Fonte: Dados da pesquisa

Conforme pode ser analisado pela Tabela 11, a partir de $n=20.000$ clientes na fila o resultado médio do tempo médio de espera na fila se aproximou do valor teórico e com $n=50.000$ os resultados médio e teórico continuaram muito próximos e a variância apresentou um resultado de 0,0000 minutos, o que representa um ótimo resultado.

Ao se comparar os resultados obtidos da quantidade necessária de clientes que chegam no sistema da fila $M / M / 2$ com a fila $M / M / 1$, percebe-se que quando 0 resultado médio das 10 replicações apresenta uma grande variância e ficam distantes do valor teórico esperado, como na fila M/M/1, a quantidade de clientes necessárias no sistema para se atingir um regime estável e ficar próximo ao teórico é consideravelmente maior do que quando os resultados apresentam uma pequena variância e um resultado médio das 10 replicações próximo ao teórico, como foi o caso da fila $\mathrm{M} / \mathrm{M} / 2$.

Ainda comparando os dois sistemas de filas simulados, $M / M / 1$ e $M / M / 2$, observa-se que para esta simulação a fila que apresentou melhores resultados foi a fila $M / M / 2$, por reduzir de forma considerável o tempo médio de espera na fila, proporcionar uma ocupação do servidor dentro dos limites aceitáveis de ocupação que são de $85 \%$ e possibilitar resultados mais próximos ao teórico com uma menor quantidade de clientes que chegam no sistema.

\section{CONCLUSÃO}

Os métodos analíticos foram aplicados as filas do tipo $\mathrm{M} / \mathrm{M} / \mathrm{s}$, com o objetivo de mostrar, de maneira intuitiva e razoavelmente simples e rápida, aos alunos de 
Engenharia, que as soluções obtidas pela abordagem de Simulação são aproximadas e são mais confiáveis quando se entende a natureza da simulação. A parte experimental utilizou o software Microsoft Excel para demonstrar questões importantes, como o tempo de aquecimento para obtenção do regime estável e as variações encontradas nos resultados das replicações da simulação.

Foram mostrados experimentos em que os valores da simulação convergiram para os valores teóricos somente após um tempo de simulação muito grande e, ainda, que as replicações sempre apresentaram variabilidade. A modelagem adaptada para as planilhas eletrônicas foi uma forma simples de visualização da dinâmica do processo de convergência, por meio do uso de gráficos. Quando se utiliza os softwares de simulação estes aspectos podem ser mascarados. Tendo em vista a variabilidade e aleatoriedade dos dados de entrada do sistema os resultados não apresentam uma precisão de 100\%, apesar de ficarem bem próximos do resultado teórico, desta forma é necessário ter um claro entendimento do funcionamento e dos objetivos do sistema real para desenvolver uma boa modelagem computacional.

O artigo não pretendeu discutir os modelos de análise de dados de simulação, que podem ser encontrados em livros tais como (LAW, 2007; CHWIF e MEDINA, 2010). Estes modelos requerem conhecimentos de Estatística, que extrapolam o que se pretendeu aqui. Deve ficar claro que, em novas aplicações, os resultados de saída devem ser tratados estatisticamente, com o apoio de ferramentas estatísticas integradas aos softwares de simulação.

A simulação é de fato útil quando não se dispõe de uma solução analítica. No caso dos sistemas de filas, são tantas as variações possíveis que a simulação pode ser uma poderosa ferramenta para se avaliar sistemas mais realistas. O uso de modelos analíticos pode trazer excessivas simplificações. A máxima da Simulação, citada por Bouzada (2010), diz que "é melhor ter uma solução aproximada para um modelo bem realista do que uma solução exata para um modelo com muitas aproximações".

\section{REFERÊNCIAS}

ABENSUR, Eder O.; BRUNSTEIN, Israel; FISCHMANN, Adalberto A. \& HO, Linda L. Tendências para o auto-atendimento bancário brasileiro: um enfoque estratégico baseado na teoria das filas. Revista de Administração Mackenzie, v.4, n.2, p. 39-59, 2003. 
ANDRADE, E. L. Introdução à pesquisa operacional: métodos e modelos para a análise de decisão. 3. ed. Rio de Janeiro: Livros Técnicos e Científicos, 2002.

ARTALEJO, J. R. \& LOPEZ-HERRERO, M. J. A simulation study of a discrete-time multiserver retrial queue with finite population. Journal of Statistical Planning and Inference, 2007. DOI: 10.1016/j.jspi.2006.04.018.

BAPTISTA, Rodrigo C. T. \& RANGEL, João J. de A. Simulação a eventos discretos de uma via semaforizada com controle automatizado em tempo real. Revista Produção Online. Florianópolis, SC, v.13, n. 1, p. 290-317, jan./mar. 2013. DOI:

http://dx.doi.org/10.14488/1676-1901.v13i1.1149

BARBOSA, Raphael A.; RODRIGUES, Thiago L.; ALMEIDA; Rafael E. P.; ESPINDOLA, Jesse R. A. \& MOREIRA, Davi F. V. Modelagem e Análise do Sistema de Filas de Caixas de Pagamento em uma Drogaria: Uma Aplicação da Teoria das Filas. XXIX Encontro Nacional de Engenharia de Produção, 2009.

BOUZADA, Marco Aurélio C. Simulação versus métodos analíticos: uma ferramenta didática na forma de discussão teórica. XXI Encontro Nacional dos Cursos de Graduação em Administração, 2010.

BRANDÃO, Rayra; GOMES, Simones A. R.; STELMASTCHUK, Juliana \& SANTOS, Yveline B. I. Teoria de Filas Aplicada a Panificadora de um Supermercado. XIX Simpósio de Engenharia de Produção, 2012.

CARNEIRO, Welington M. Experimentação em um Sistema de Filas Utilizando a Simulação Computacional: Um Estudo de Caso. XXVIII Encontro Nacional de Engenharia de Produção, 2008.

CHUNG, Christopher A. Simulation modeling handbook: a practical approach. Florida: CRC Press, 2004. Disponível em: <https://books.google.com.br/books?hl=pt-

$B R \& \mid r=\& i d=J h V 6 R y 6 U M D Q C \& o i=f n d \& p g=P P 1 \& d q=$ Simulation+modeling + handbook:+a+pra ctical+approach\&ots=JiWxmR40QI\&sig=M-Dg-

ILKpZuutPenAj3NpWKaxGA\#v=onepage \&q=Simulation\%20modeling\%20handbook\%3A\%2 0a\%20practical\%20approach\&f=false> Acesso em: 15 set 2014

CHWIF, Leonardo \& MEDINA, Afonso C. Modelagem e Simulação de Eventos Discretos: Teoria e Aplicações. 3. Ed. rev. São Paulo: Ed. do Autor, 2010.

EVANS, James. R. Spreadsheets as a Tool for Teaching Simulation. College of Business Administration University of Cincinnati. Cincinnati, Ohio 45221, USA, 2000.

FITZSIMMONS, James A. \& FITZSIMMONS, Mona J. Administração de Serviços: operações, estratégia e tecnologia da informação. 6. Ed. Porto Alegre: Ed. Bookman, 2010.

FOGLIATTI, Maria C.; MATTOS, Néli M. C. Teoria de Filas. Rio de Janeiro: Interciência, 2007.

FREITAS FILHO, Paulo J. Introdução à Modelagem e Simulação de Sistemas com Aplicações em Arena. 2. ed. Florianópolis: Visual Books, 2008.

HILLIER, Frederick S. \& LIEBERMAN, Gerald J. Introdução à Pesquisa Operacional. 9.ed. Porto Alegre: AMGH, 2013. 
INGOLFSSON, Armann., GROSSMAN Jr, Thomas. A. Graphical Spreadsheet Simulation of Queues. INFORMS Transactions on Education 2(2):27-39, 2002. DOI:

http://dx.doi.org/10.1287/ited.2.2.27.

LAW, Averill M. Simulation Modeling \& Analysis. 4. ed. Boston: Ed. McGraw-Hill, 2007.

LIMA, Danilo F. S. de; ALCANTARA, Paulo G. de F.; SANTOS, Luciano C.; SILVA, Liane M. F. e \& SILVA, Ricardo M. da. Mapeamento do Fluxo de Valor E Simulação para Implementação de Práticas Lean em uma Empresa Calçadista. Revista Produção Online. Florianópolis, SC, v. 16, n. 1, p. 366-392, jan./mar. 2016. DOI:

http://dx.doi.org/10.14488/1676-1901.v16i1.2183.

NORATO, Hélida G. \& DUARTE, Anderson R. Uma proposta para a detecção do adequado período de aquecimento em modelos de simulação. Gestão da Produção, Operações e Sistemas. Ano 6, n.4, p. 11-26, 2011.

PEREIRA, Carla R. \& COSTA, Miguel A. B. da. Um Modelo de Simulação de Sistemas Aplicado à Programação da Produção de um Frigorífico de Peixe. Revista Produção Online. Florianópolis, SC, v.12, n. 4, p. 972-1001, out./dez. 2012. DOI:

http://dx.doi.org/10.14488/1676-1901.v12i4.994

PERIN, Clóvis F. Introdução à simulação de sistemas. Campinas, Ed. da Unicamp, 1995.

PRADO, Darci S. Usando o ARENA em Simulação. 3. ed. Belo Horizonte: Ed. INDG Tecnologia e Serviços Ltda., 2008.

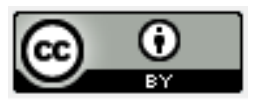

Artigo recebido em 12/02/2016 e aceito para publicação em 10/03/2016 DOI: http://dx.doi.org/ 10.14488/1676-1901.v17i1.2360 